\title{
Dimensionamento otimizado da seção transversal de pilares em concreto armado submetidos à flexocompressão
}

\author{
Rodrigo Bordignon e Moacir Kripka ${ }^{(1)}$
}

\begin{abstract}
Resumo
Este trabalho apresenta o desenvolvimento e a implementação computacional de uma formulação matemática visando a otimizaçào das seções transversais de pilares em concreto armado sujeitos à flexocompressão reta, com base na norma técnica brasileira ABNT NBR 6118/07. Com o objetivo de minimizar o custo total da seção foi empregado o método Simulated Annealing, sendo que a tanto a quantidade e Bitola das armaduras quanto as dimensões da seção de concreto foram consideradas como variáveis discretas. Os resultados obtidos foram comparados tanto com o procedimento convencional de dimensionamento por ábacos como com outros métodos de otimização.
\end{abstract}

Palauras-chave: Otimização. Pilares. Concreto Armado. Simulated Annealing.

(1) Programa de Pós-Graduação em Engenharia Civil e Ambiental - PPGEng. Universidade de Passo Fundo - Passo Fundo, RS. E-mail: bordignon@upf.br, mkripka@upf.br 


\section{Introdução}

A busca por um projeto ótimo de uma estrutura ou obra de engenharia, que atenda à máxima funcionalidade e segurança com o mínimo custo e, mais do que nunca, mínimo impacto ambiental, sempre foi o objetivo almejado pela maioria dos engenheiros projetistas. Atualmente, com o avanço da tecnologia é possível investigar um maior número de variáveis em um projeto, diminuindo as simplificações e tornando o modelo matemático cada vez mais representativo do estado real.

Sendo muitas as soluções possíveis, a escolha da mais adequada pode ser facilitada com a implementação de técnicas matemáticas de otimização. Atualmente, há várias ferramentas de análise e dimensionamento de estruturas, mas não é comum a incorporação de módulos de otimização matemática para que essas ferramentas possam analisar e dimensionar de forma otimizada uma estrutura.

No campo da engenharia estrutural, seja em concreto armado, aço ou madeira, o emprego de técnicas matemáticas de otimização geralmente objetiva determinar as dimensões da seção transversal dos elementos que minimize o custo ou o peso total da estrutura, atendendo aos estados limites preconizados por normas técnicas. Relativamente a estruturas de concreto armado, é usual a obtenção das dimensões da seção de concreto e das áreas das armaduras e das formas. Neste sentido, destacam-se os estudos realizados por Zielinski, Long e Troitsky (1995), que apresentam um procedimento para otimização de pilares em concreto armado; de Argolo (2000), que desenvolveu um estudo de otimização de seções de concreto armado submetidas à flexocompressão reta; de Rodrigues Júnior (2005), que estudou uma formulação para o projeto ótimo de pilares de edifícios altos de concreto armado e de Martínez (2007), que comparou diversos algoritmos de otimização que permitiam obter o dimensionamento de pilares retangulares de seção vazada de concreto armado de viadutos rodoviários e ferroviários de diferentes alturas e vãos. Um extenso estudo sobre aplicações de técnicas de otimização a estruturas pode ser encontrado em Medeiros e Kripka (2012).

\section{Fundamentos da Otimização Matemática}

Algumas vantagens do uso da otimização matemática são apresentadas por Vanderplaats (1984), das quais a redução do tempo de projeto é uma das principais, uma vez que a busca por melhores soluções é efetuada de forma sistematizada, e não por tentativas. Além disso, a possibilidade da consideração simultânea de um grande número de variáveis e de restrições pode conduzir a soluções não convencionais.

A otimização matemática de um problema pode ser alcançada ao se encontrar a melhor das soluções possíveis com o auxílio de um algoritmo apropriado, levando em conta as variáveis de projeto e as restrições.

De forma geral, a formulação matemática básica de um problema de otimização pode ser descrita como (VANDERPLAATS, 1984): 
Minimizar:

$$
f\left(x_{i}\right) \quad i=1, n
$$

Sujeito a:

$$
\begin{aligned}
& g_{j}\left(x_{i}\right) \leq 0 \quad j=1, m \\
& h_{k}\left(x_{i}\right)=0 \quad k=1, l \\
& x_{i}{ }^{l} \leq x_{i} \leq x_{i}{ }^{u}
\end{aligned}
$$

onde $f$ é a função objetivo e $x=\left(x_{1}, x_{2}, \ldots x_{n}\right)^{T}$ consiste no vetor das variáveis de projeto, de dimensão $n$. As demais funções são as chamadas restrições do problema (restrições de desigualdade $g$, de igualdade $h$ e espaço de possíveis valores de $x$ ). $\mathrm{Na}$ equação (4), os índices $l$ e $u$ indicam, respectivamente, os limites inferiores e superiores para as variáveis.

Desde a década de 1970, tem aumentado o interesse por métodos heurísticos para a resolução de problemas complexos de otimização, tais como aqueles envolvendo funções descontínuas ou com vários pontos de mínimo, para os quais os métodos tradicionais se mostram pouco eficientes. Os métodos heurísticos efetuam a comparação do valor da função em diversos pontos, sem a consideração de informações de derivadas ou aproximações de derivadas. Em contrapartida, acarretam um maior número de iterações.

Os métodos heurísticos mais largamente empregados foram inspirados no comportamento da natureza, com base na física e na biologia. Dentre estes, o mais difundido é o Método dos Algorítmos Genéticos, desenvolvido por Goldberg (1989) em analogia ao processo evolutivo. O método trabalha com uma população de indivíduos (ou soluções) os quais, segundo a aptidão (valor da função), sofrem cruzamentos e mutações, gerando novas gerações.

O presente trabalho objetiva apresentar a aplicação de um método heurístico de otimização, o método Simulated Annealing, na minimização do custo da seção de pilares retangulares em concreto armado submetidos à flexocompressão reta. Com esta finalidade foram seguidos os preceitos da norma brasileira ABNT NBR 6118 - Projeto de estruturas de concreto - Procedimento (2007).

Nos itens seguintes deste trabalho são descritos o método de otimização, o procedimento para verificação da capacidade resistente das seções e a formulação desenvolvida. Na sequência são apresentados alguns resultados obtidos a partir da implementação da formulação, bem como as conclusões decorrentes.

\subsection{Método Simulated Annealing}

As técnicas de otimização normalmente empregadas são baseadas em estratégias descendentes. Nestas, partindo de uma solução inicial, uma nova solução é gerada e seu valor comparado ao valor inicial. Caso se verifique uma redução no valor da função, a solução correspondente a este novo valor é adotada como a solução atual, e o procedimento repetido até que nenhum melhoramento significativo possa ser obtido. A solução final, dependendo das características das funções envolvidas, pode corresponder à melhor solução nas vizinhanças, mas não necessariamente no espaço de busca. Uma 
estratégia usual para tentar melhorar a solução obtida consiste em se efetuar a análise de um mesmo problema a partir de diversas soluções iniciais, supondo-se que, caso a mesma solução seja obtida a partir destes pontos, esta corresponderá ao mínimo global.

O Método Simulated Annealing emprega uma estratégia diferente, ao tentar evitar a convergência para um ponto de mínimo local aceitando também, segundo um critério específico, soluções que acarretem em aumento no valor da função. O Simulated Annealing é um método heurístico desenvolvido por Kirkpatrick, Gelatt e Vecchi (1983), fundamentado na simulação do processo mecânico de recozimento de metais. Quando um metal é aquecido a altas temperaturas, provocando a fusão, esse processo é chamado de recozimento (annealing) e os átomos se movimentam livremente. A solidificação se dá pelo resfriamento lento e controlado e leva a que esses átomos se organizem numa configuração ordenada e estável, formando uma estrutura uniforme e com energia mínima, tendo como resultado uma redução de defeitos no material. Caso o metal seja resfriado de forma brusca, a microestrutura tende a um estado instável.

Metrópolis et al. (1953) apresentaram um algoritmo para modelagem do processo de recozimento de metais, simulando as mudanças de energia em um sistema de partículas à medida que a temperatura diminui até um estado estável. A aceitação desse tipo de solução depende de uma probabilidade conhecida como "critério de Metrópolis", calculada pela função:

$$
p(\Delta E)=\exp \left(\frac{-\Delta E . K}{T}\right)
$$

onde $T$ é a temperatura do corpo e $K$, a constante de Boltzmann.

Na simulação do recozimento como uma técnica de otimização, a função objetivo corresponde à energia do sólido. Da mesma maneira que no método original de recozimento na termodinâmica, o processo inicia com um alto valor de $T$, para o qual uma nova solução é gerada e $T$ passa a ser um parâmetro de controle, e a constante de Boltzmann não tem analogia num problema de otimização, sendo eliminada. $\mathrm{O}$ aceite dessa nova solução dar-se-á automaticamente caso acarrete redução no valor da função; em oposição, sendo o novo valor da função maior que o anterior, o aceite dar-se-á por um critério probabilístico, sendo a função de aceite:

$$
p=\exp \left(\frac{-\Delta f}{T}\right)
$$

Gera-se um número randômico " $r$ " a partir de uma distribuição uniforme no intervalo $[0,1]$. Se este número for menor ou igual a " $p$ ", aceita-se a solução; em caso contrário, a solução é rejeitada. 


\section{Processo iterativo de verificação da capacidade resistente}

O processo iterativo inicia a partir do conhecimento do esforço normal atuante de cálculo $\left(\mathrm{N}_{\mathrm{sd}}\right)$, do momento fletor de cálculo $\left(\mathrm{M}_{\mathrm{sd}}\right)$ e de uma seção transversal com arranjos e diâmetros das barras conhecidos.

Os esforços atuantes de cálculo $\mathrm{N}_{\mathrm{sd}}$ e $\mathrm{M}_{\mathrm{sd}}$ são obtidos pela multiplicação dos esforços característicos pelos respectivos coeficientes parciais de segurança, fornecidos pela norma ABNT NBR 8681 (2004) para as diferentes ações e combinações envolvidas no projeto.

Os esforços resistentes $\mathrm{N}_{\mathrm{rd}}$ e $\mathrm{M}_{\mathrm{rd}}$ obedecem à mesma convenção de sinais adotada para os solicitantes e, de acordo com a descrição da configuração deformada, são obtidos pelas seguintes equações de equilíbrio:

$$
\begin{gathered}
N_{r d}=\int_{A c} \sigma_{c d} \cdot d A_{c}+\sum_{i=1}^{n} A_{s i} \cdot \sigma_{s d i} \\
M_{r d}=\int_{A c} \sigma_{c d} \cdot y_{c} \cdot d A_{c}+\sum_{i=1}^{n} A_{s i} \cdot \sigma_{s d i} \cdot y_{s i}
\end{gathered}
$$

onde:

$\mathrm{N}_{\mathrm{rd}}$ é o esforço normal resistente de cálculo;

$\mathrm{M}_{\mathrm{rd}}$ é o momento resistente de cálculo;

$\sigma_{c d}$ é a tensão na área de concreto comprimida $A_{c}$;

$\sigma_{\mathrm{sdi}}$ é a tensão na área de aço $\mathrm{A}_{\mathrm{si}}$;

$\mathrm{y}_{\mathrm{c}}$ é a distância do centro da área de concreto comprimida, em relação ao centro de gravidade da seção;

$\mathrm{y}_{\mathrm{si}}$ é a distância da barra de aço $i$, em relação ao centro de gravidade da seção.

O esforço normal resistente de cálculo $\mathrm{N}_{\mathrm{rd}}$ deve ser no mínimo igual ao esforço normal solicitante $\mathrm{N}_{\mathrm{sd}}$, aplicado a uma seção transversal com armaduras conhecidas. $\mathrm{O}$ valor de $\mathrm{N}_{\mathrm{sd}}$ é fixo, enquanto $\mathrm{N}_{\mathrm{rd}}$ varia em função da profundidade $\mathrm{x}_{0}$ da linha neutra, que é a única incógnita presente na equação 9:

$$
f\left(x_{0}\right)=N_{s d}-A_{c c} \cdot \sigma_{c d}-\sum_{i=1}^{n} A_{s i} \cdot \sigma_{s d i}
$$

A Figura 1 ilustra uma seção genérica submetida a flexocompressão oblíqua. No caso de flexocompressão reta, como abordado no presente trabalho, a flexão se dá apenas em torno do eixo $\mathrm{X}$ (ângulo $\alpha=180^{\circ}$ ), sendo portanto a linha neutra LN também paralela a esse eixo. 


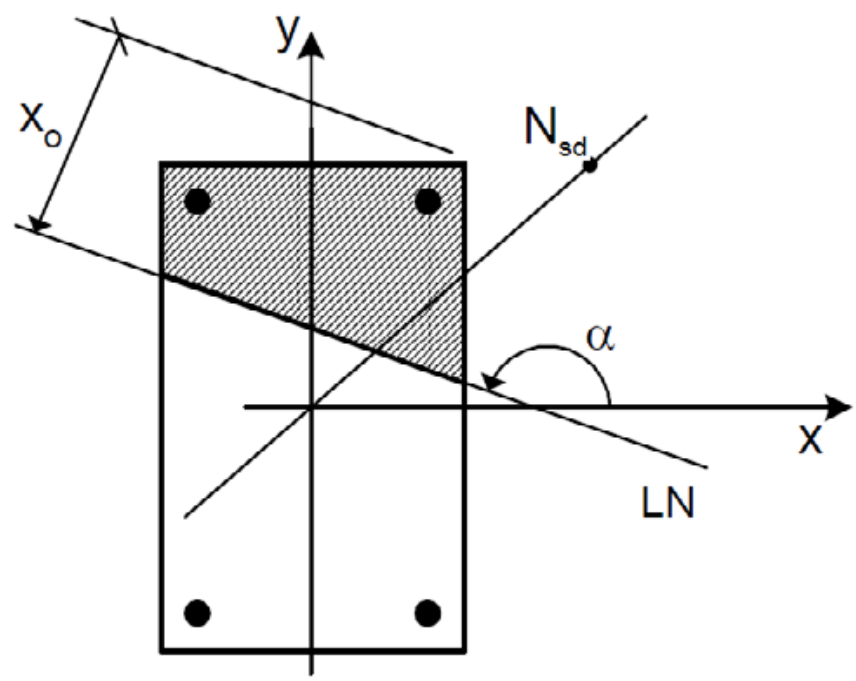

Figura 1: Caso geral (flexocompressão oblíqua)

Fazendo $f\left(x_{0}\right)=0$ na equação (9), tem-se que a solução, situada no intervalo $[0, \infty]$ abrangendo todos os domínios da flexocompressão, pode ser determinada por algum processo iterativo. Neste trabalho, a solução da equação, que fornece a posição da linha neutra, foi efetuada com o emprego do Método da Seção Áurea, um método de busca unidimensional que se caracteriza por necessitar somente do cálculo dos valores da função em determinados pontos (sem o conhecimento de suas derivadas), de modo que o intervalo de valores é reduzido até a convergência para um valor único, conforme uma tolerância estabelecida.

Conhecida a profundidade $\mathrm{x}_{0}$ da linha neutra, as equações de equilíbrio (7) e (8) tomam a seguinte forma:

$$
\begin{gathered}
N_{r d}=A_{c c} \cdot \sigma_{c d}+\sum_{i=1}^{n} A_{s i} \cdot \sigma_{s d i} \\
M_{r d}=S_{c} \cdot \sigma_{c d}+\sum_{i=1}^{n} A_{s i} \cdot \sigma_{s d i} \cdot y_{s i}
\end{gathered}
$$

onde:

$\mathrm{A}_{\mathrm{cc}}$ representa a área de concreto comprimida;

$\mathrm{S}_{\mathrm{c}}$ representa o momento estático da parte de concreto comprimida da seção. 


\section{Formulação do Problema de Otimização}

A otimização de uma seção em concreto armado submetida à flexocompressão reta consiste em um problema multidimensional. Isso se deve a vários fatores relacionados à topologia da seção, restrições normativas, entre outros.

Considerando uma seção transversal retangular qualquer, o objetivo do dimensionamento ótimo é obter uma configuração desta que seja capaz de produzir esforços resistentes $\left(\mathrm{N}_{\mathrm{rd}}\right.$ e $\mathrm{M}_{\mathrm{rd}}$ ), iguais ou superiores aos esforços solicitantes $\left(\mathrm{N}_{\mathrm{sd}}\right.$ e $\mathrm{M}_{\mathrm{sd}}$ ), com o mínimo custo.

A formulação do problema de otimização parte da consideração de alguns parâmetros de entrada, definidos previamente e que representam, basicamente, os esforços atuantes no elemento e as características e custos dos materiais. Esses parâmetros de projeto são informados e assumem valores fixos, ou seja, não se alteram durante o processo de otimização e são representados por:

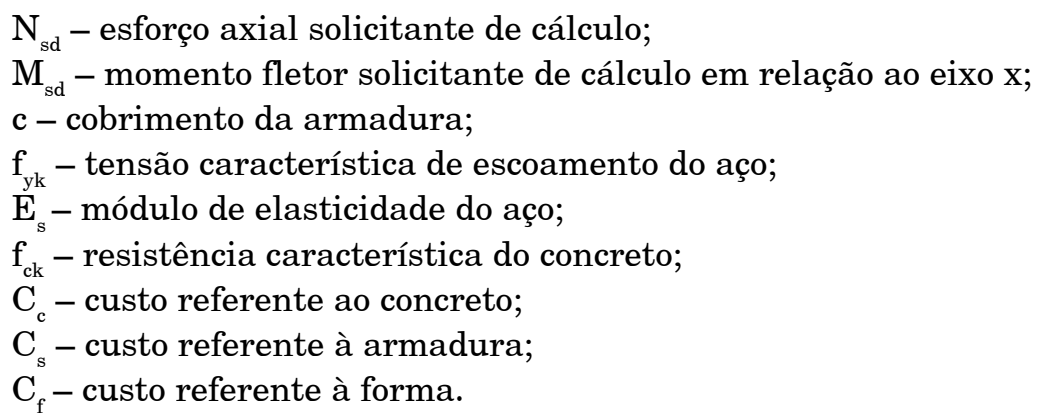

As variáveis de projeto $\left(\mathrm{x}_{\mathrm{i}}\right)$ são os valores relacionados ao dimensionamento da seção transversal do concreto e das áreas, quantidade e disposição das armaduras. Para cada conjunto de valores é gerada uma configuração com determinada capacidade resistente e que deve atender às prescrições de norma. A figura (2) ilustra as variáveis de projeto, sendo:

$\mathrm{x}_{1}$ - largura da seção transversal (b);

$\mathrm{x}_{2}$ - altura da seção transversal (h);

$\mathrm{x}_{3}$-diâmetro de cada uma das quatro barras dos cantos;

$\mathrm{x}_{4}$ - número de barras em cada camada na dimensão $\mathrm{x}_{1}$;

$\mathrm{x}_{5}$ - diâmetro das barras paralelas a $\mathrm{x}_{1}$;

$\mathrm{x}_{6}$ - número de camadas com duas barras paralelas a $\mathrm{x}_{2}$;

$\mathrm{x}_{7}$ - diâmetro das barras nas camadas paralelas a $\mathrm{x}_{2}$.

Exemplificando, para uma seção transversal composta por barras apenas nas extremidades, os valores de $\mathrm{x}_{4}$ a $\mathrm{x}_{7}$ são nulos. 


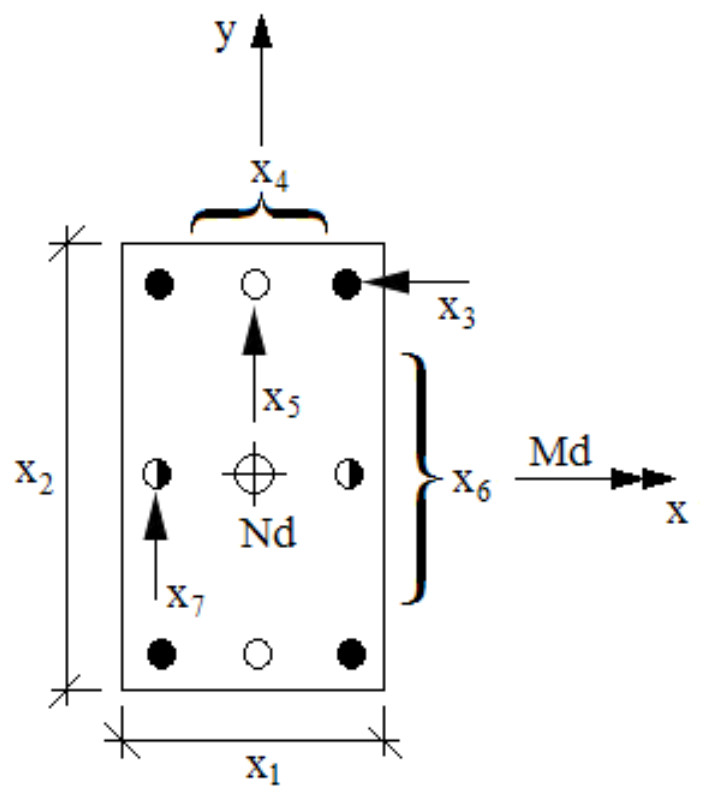

Figura 2: Variáveis de projeto

A função objetivo é determinada em decorrência dos valores atribuídos às variáveis de projeto, e representa o custo da solução a partir dos quantitativos e dos custos unitários de cada material, sendo expressão da seguinte forma:

$$
\operatorname{minimizar} F(x)=\left(x_{1} \cdot x_{2}\right) \cdot C_{c}+\left(4 \cdot x_{3}+2 \cdot x_{4} \cdot x_{5}+2 \cdot x_{6} \cdot x_{7}\right) \cdot(\pi / 4) \cdot y_{s} \cdot C_{s}+2 \cdot\left(x_{1}+x_{2}\right) \cdot C_{f}
$$

A primeira parcela da função representa o custo do concreto, em que $\mathrm{C}_{\mathrm{c}}$ é o custo por unidade de volume; a segunda representa o custo da armadura longitudinal, sendo $\mathrm{C}_{\mathrm{s}}$ o respectivo custo por unidade de massa, e $\square_{s}$ o peso específico do aço; a última parcela representa o custo relativo à forma, onde $\mathrm{C}_{\mathrm{f}}$ é o custo por unidade de área. Todos os custos fornecem um valor relativo para cada unidade de comprimento do elemento otimizado.

No processo de minimizar da função custo, deve-se também respeitar o conjunto de restrições impostas ao problema. Basicamente, as restrições são relativas aos critérios de resistência e restrições construtivas, como citado anteriormente.

Todas as variáveis de projeto devem atender aos preceitos da norma ABNT NBR 6118 (2007) com referência às limitações de dimensões, espaçamentos, taxas geométricas de armaduras. Logo: 
$\mathrm{x}_{1}$ e $\mathrm{x}_{2}$ - são variáveis discretas. No presente trabalho, optou-se por considerá-las com incremento na dimensão de um em um centímetro, representadas pelos intervalos:

$$
\begin{aligned}
& \mathrm{x}_{1} \in[19,20, \ldots, 200] \\
& \mathrm{x}_{2} \in[19,20, \ldots, 1000]
\end{aligned}
$$

sendo os valores superiores suficientemente grandes, de modo a não interferirem na solução ótima. Pelo mesmo motivo, as variáveis $\mathrm{x}_{4}$ e $\mathrm{x}_{6}$ (número de barras nas duas camadas paralelas a $\mathrm{x}_{1}$ e número de camadas com duas barras paralelas a $\mathrm{x}_{2}$, respectivamente, podem assumir valores inteiros entre 0 e 10 .

$\mathrm{x}_{3}, \mathrm{x}_{5}$ e $\mathrm{x}_{7}$ - as variáveis que representam as barras longitudinais ficam limitadas a valores reais, e restritas aos seguintes diâmetros (em $\mathrm{mm})$ :

$$
\mathrm{x}_{3,} \mathrm{x}_{5} \mathrm{e}_{7} \in[10.0,12.5,16.0,20.0,22.0,25.0,32.0,40.0]
$$

Portanto, as restrições do problema podem ser reescritas na forma normalizada, visando a um melhor tratamento computacional, da seguinte forma:

$$
\begin{gathered}
g_{1}=1-N_{r d} / N_{s d} \leq 0 \\
g_{2}=1-M_{r d} / M_{s d} \leq 0 \\
g_{3}=1-b / b_{\text {min }}=1-x_{1} / 19 \leq 0 \\
g_{4}=1-b_{\text {máx }} / b=1-200 / x_{1} \leq 0 \\
g_{5}=1-h / h_{\text {min }}=1-x_{2} / 19 \leq 0 \\
g_{6}=1-h_{\text {max }} / h=1-1000 / x_{2} \leq 0 \\
g_{7}=1-5 b / h=1-5 x_{1} / x_{2} \leq 0 \\
g_{8}=1-e / e_{\text {min }} \leq 0 \\
g_{9}=1-e_{\text {max }} / e \leq 0 \\
g_{10}=1-\rho / \rho_{\text {min }} \leq 0 \\
g_{11}=1-\rho_{\text {máx }} / \rho \leq 0
\end{gathered}
$$


A restrição relativa à dimensão mínima da seção de concreto não precisa ser considerada de forma explícita, pois já é automaticamente atendida em função dos valores mínimos estipulados para as dimensões das faces (variáveis $\mathrm{x}_{1}$ e $\mathrm{x}_{2}$ ).

Para o tratamento das restrições é empregada a técnica das funções de penalização, no qual os problemas com restrição são transformados em problemas irrestritos, acrescentando-se uma função de penalidade $P(x)$, que representa o conjunto das restrições. Assim, o custo de uma solução que não atende integralmente às restrições do problema é artificialmente aumentado.

De forma geral, nas simulações efetuadas, partiu-se de mais de uma solução inicial, obtendo-se a convergência para uma única solução otimizada. O método Simulated Annealing trabalha com parâmetros em analogia ao processo de recozimento, e que podem ser obtidos por experimentos ou com base em indicação da literatura. No caso do problema aqui abordado, foram adotados os seguintes parâmetors: temperatura inicial $(T)=1000$, redutor de temperatura $(\square)=0,98$, fator de penalização $(r)=1000$ e critério de parada $=\mathrm{T}_{\mathrm{k}} / \mathrm{T} \leq 0,0001$ (relação entre a temperatura inicial e a atual).

\section{Exemplos}

A formulação proposta, descrita anteriomente, foi implementada a partir de um programa base desenvolvido anteriormente por Kripka (2003), para a otimização com o emprego do método Simulated Annealing. A esse programa foi associada uma rotina para a verificação da capacidade resistente de pilares submetidos à flexocompressão reta, utilizando-se a linguagem de programação Fortran.

Os valores unitários da armadura e forma para a composição do custo foram extraídos do Guia da Construção (2010), onde estão inclusos os materiais, mão de obra, equipamentos e leis sociais praticados no Rio Grande do Sul no mês de setembro de 2010. Para o concreto, os valores seguem os praticados na região, referentes ao mesmo mês.

\subsection{Exemplo I}

Dois exemplos de dimensionamento convencional foram extraídos de Araújo (2003), onde para um mesmo par de esforços são apresentadas duas configurações de disposição da armadura longitudinal, a partir de dimensões da seção transversal predefinidas. Na Tabela 1 são apresentados os valores unitários dos materiais e o par de esforços utilizado neste exemplo.

Tabela 1: Custos unitários e esforços do exemplo I

\begin{tabular}{c|c|c|c|c}
\hline $\mathrm{C}_{\mathrm{c}}\left(\mathrm{R} \$ / \mathrm{m}^{3}\right)$ & $\mathrm{C}_{\mathrm{s}}(\mathrm{R} \$ / \mathrm{kg})$ & $\mathrm{C}_{\mathrm{f}}\left(\mathrm{R} \$ / \mathrm{m}^{2}\right)$ & \multicolumn{2}{|c}{ Esforços Solicitantes } \\
\hline $20 \mathrm{MPa}$ & $500 \mathrm{MPa}$ & - & $\mathrm{N}_{\mathrm{sd}}(\mathrm{kN})$ & $\mathrm{M}_{\mathrm{sd}}(\mathrm{kN} . \mathrm{cm})$ \\
245,00 & 6,56 & 42,36 & 574 & 14350 \\
\hline
\end{tabular}


O primeiro modelo de dimensionamento corresponde a uma seção transversal retangular com dimensões de $20 \times 40 \mathrm{~cm}$ e composta de duas camadas de armadura, cuja área de aço necessária encontrada a partir do uso de tabelas foi de $15,7 \mathrm{~cm}^{2}$. A composição final da seção se dá a partir de diâmetros comerciais, resultando em quatro barras de aço de $16 \mathrm{~mm}$ de diâmetro em cada camada, gerando uma área de aço final de 16,08 $\mathrm{cm}^{2}$ na seção.

Em outro modelo, a mesma seção transversal de 20 x $40 \mathrm{~cm}$ e submetida aos mesmos esforços é dimensionada empregando-se o mesmo método do primeiro, somente alterando de dois para quatro o número de camadas. $\mathrm{O}$ resultado encontrado são duas barras de aço de $20 \mathrm{~mm}$ de diâmetro em cada uma das camadas, ficando a seção transversal composta com uma área de aço total de $25,13 \mathrm{~cm}^{2}$.

Neste estudo, duas seções foram geradas de forma otimizada a fim de verificar a eficiência do procedimento proposto, ambas capazes de resistir aos mesmos esforços, empregando os mesmos materiais e seguindo as restrições impostas pela norma ABNT NBR 6118 (2007). Numa delas, com dimensões da seção transversal fixas de 20 x 40 $\mathrm{cm}$, a armadura longitudinal foi otimizada pelo método Simulated Annealing (S.A.). Neste caso, a área de aço ótima obtida se iguala ao valor mínimo encontrado por Araújo (2003) com o emprego de tabelas. Porém, como neste estudo a armadura longitudinal pode ser composta por até três bitolas distintas, essa combinação possibilitou uma redução de $0,38 \mathrm{~cm}^{2}$ na área de aço final da seção transversal.

$\mathrm{O}$ segundo modelo dimensionado pelo método convencional apresenta um custo $30,38 \%$ superior em comparação com a seção do primeiro modelo gerada pelo mesmo método. Quando as seções ótimas são comparadas a do primeiro modelo, a economia é de $1,19 \%$ no custo total, chegando a $20,50 \%$ quando a seção ótima é gerada de forma livre (variando também as dimensões da seção de concreto). A Figura 3 apresenta as configurações correspondentes às seções dimensionadas da forma convencional (ábacos de interação). Já a Figura 4 ilustra as configurações otimizadas.

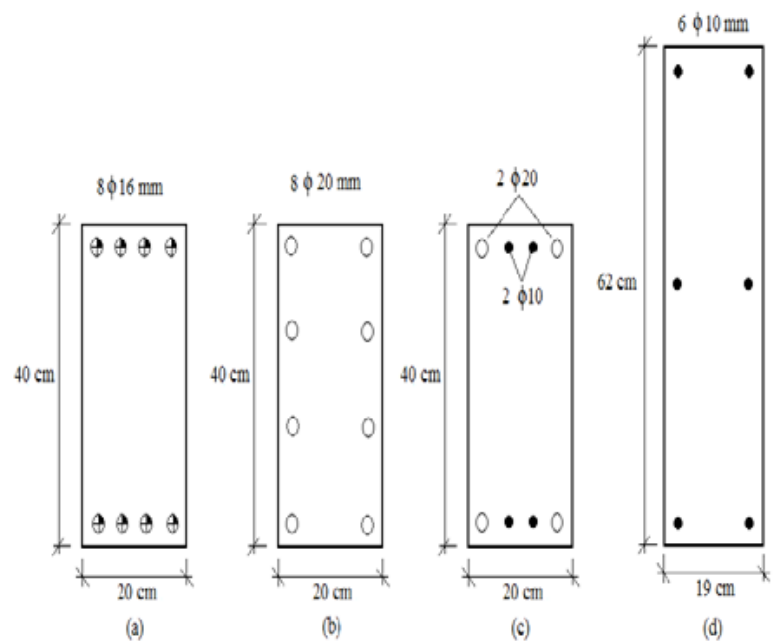

Figura 3: Detalhamento das seções por dimensionamento convencional 


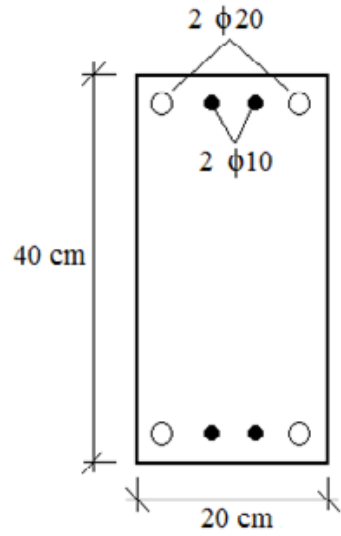

(a)

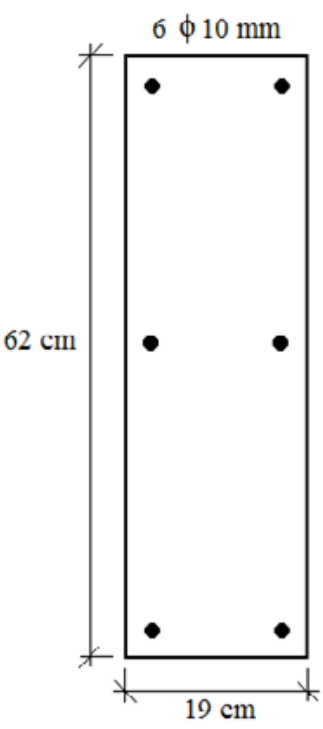

(b)

Figura 4: Detalhamento das seções ótimas

\subsection{Exemplo II}

Este exemplo de flexocompressão reta foi estudado por Argolo (2000), cuja análise foi baseada na norma ABNT NBR 6118 (1980). O autor comparou os custos a partir de uma seção transversal de $30 \times 70 \mathrm{~cm}$, inicialmente dimensionada com o auxílio de ábacos de iteração para três diferentes pares de esforços $\mathrm{N}_{\mathrm{sd}}$ e $\mathrm{M}_{\mathrm{sd}}$, todos sobre a mesma envoltória, resultando em uma mesma área de aço para as três situações. Logo, essas mesmas seções foram submetidas à otimização da armadura longitudinal, com dimensões da seção transversal fixas, empregando o método dos Algoritmos Genéticos (A.G.).

A composição do custo unitário dos materiais e forma utilizados neste exemplo é relativa ao mês de março de 2000, com os mesmos valores utilizados por Argolo (2000), como mostra a Tabela 2. Da mesma maneira, o par de esforços utilizados neste exemplo representa o segundo modelo estudado pelo autor e será comparado por conter uma distribuição simétrica da armadura longitudinal na seção, semelhante ao adotado neste estudo.

Tabela 2: Custos unitários e esforços do exemplo II

\begin{tabular}{c|c|c|c|c}
\hline $\mathrm{C}_{\mathrm{c}}\left(\mathrm{R} \$ / \mathrm{m}^{3}\right)$ & $\mathrm{C}_{\mathrm{s}}(\mathrm{R} \$ / \mathrm{kg})$ & $\mathrm{C}_{\mathrm{f}}\left(\mathrm{R} \$ / \mathrm{m}^{2}\right)$ & \multicolumn{2}{|c}{ Esforços Solicitantes } \\
\hline $25 \mathrm{Mpa}$ & $500 \mathrm{Mpa}$ & - & $\mathrm{N}_{\mathrm{sd}}(\mathrm{kN})$ & $\mathrm{M}_{\mathrm{sd}}(\mathrm{kN} . \mathrm{cm})$ \\
125,00 & 1,27 & 16,49 & 2142,86 & 37500 \\
\hline
\end{tabular}


A partir deste par de esforços, duas seções são geradas empregando o método Simulated Annealing, seguindo as restrições impostas pela norma ABNT NBR 6118 (2007): uma com dimensões fixas de 30 x $70 \mathrm{~cm}$ e em que somente a armadura longitudinal é otimizada; outra na qual tanto a armadura quanto as dimensões são otimizadas pelo algoritmo.

A seção de dimensões fixas de $30 \times 70 \mathrm{~cm}$ cuja armadura passou pelo processo de otimização pelo método Simulated Annealing apresenta um custo 3,35\% inferior quando comparada à mesma seção otimizada pelo método do Algoritmo Genético estudado por Argolo (2000). Quando a seção ótima, gerada de forma livre pelo processo de otimização deste estudo é comparada ao método utilizado pelo autor em referência, a economia chega a $6,85 \%$.

As figuras 5 e 6 ilustram, respectivamente as configurações obtidas por Argolo (algorítmos genéticos) e pelo presente estudo (Simulated Annealing).

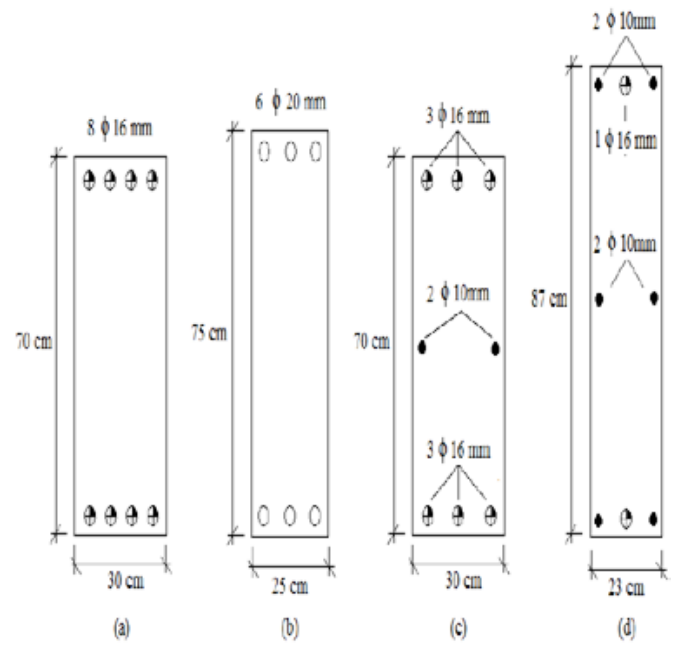

Figura 5: Detalhamento das seções pelo método do Algoritmo Genético 


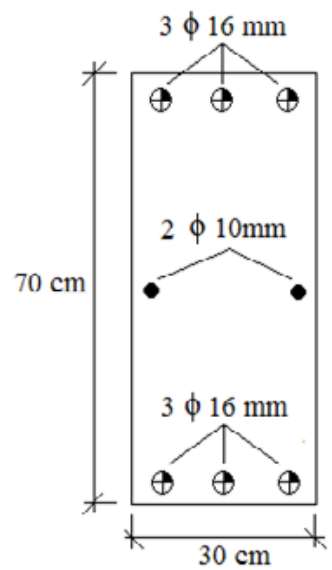

(a)

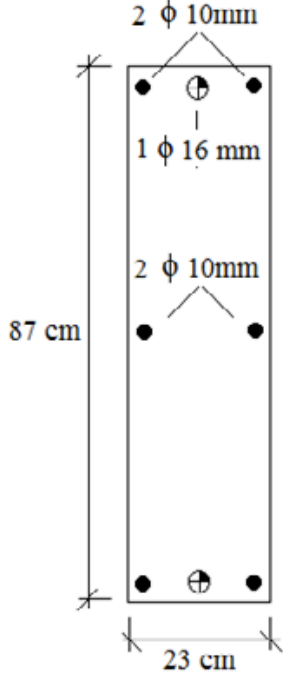

(b)

Figura 6: Detalhamento das seções pelo método do Simulated Annealing

Quando as seções ótimas geradas pelo método Simulated Annealing são comparadas ao dimensionamento realizado por Argolo (2000) com o uso de ábaco de iteração, a redução no custo total é de $29,10 \%$ para a seção com armadura otimizada e dimensões fixas de 30 x $70 \mathrm{~cm}$ e de 31,82\% para seção ótima gerada de forma livre.

Cabe salientar que neste exemplo a análise da seção realizada por Argolo (2000), tanto pelo método prático quanto pelo ótimo, não leva em conta algumas disposições construtivas exigidas pela norma ABNT NBR 6118 (2007) para pilares de concreto armado relativas aos espaçamentos máximos entre armaduras longitudinais. Desta forma, as soluções apresentadas não seriam factíveis sob a ótica da formulação empregada no presente trabalho.

\subsection{Exemplo III}

Neste exemplo, Zielinski, Long e Troitsky (1995) estudaram um caso de flexocompressão reta com o objetivo de determinar a área da seção transversal de concreto e a área de aço necessária para resistir aos esforços aplicados. A análise da seção foi feita de acordo com a norma canadense CSA CAN3-A23.3-M84.

Os custos dos materiais empregados no exemplo, juntamente com os esforços, são apresentados na Tabela 3.

Tabela 3 - Custos e esforços do exemplo III

\begin{tabular}{c|c|c|c|c}
\hline $\mathrm{C}_{\mathrm{c}}\left(\$ / \mathrm{m}^{3}\right)$ & $\mathrm{C}_{\mathrm{s}}(\$ / \mathrm{kg})$ & $\mathrm{C}_{\mathrm{f}}\left(\$ / \mathrm{m}^{2}\right)$ & \multicolumn{2}{|c}{ Esforços Solicitantes } \\
\hline $25 \mathrm{MPa}$ & $400 \mathrm{MPa}$ & - & $\mathrm{N}_{\mathrm{sd}}(\mathrm{kN})$ & $\mathrm{M}_{\mathrm{sd}}(\mathrm{kN} . \mathrm{cm})$ \\
110,00 & 2,10 & 27,00 & 2460 & 44300 \\
\hline
\end{tabular}


O dimensionamento ótimo, baseado em programação matemática (P.M.) e utilizando o Método de Powell, sugerido pelos autores, corresponde a uma seção retangular de dimensões de 39,57 x 68,36 cm e uma área de aço da armadura longitudinal da seção de $27,46 \mathrm{~cm}^{2}$.

Esta mesma seção foi simplificada para valores práticos de execução, assumindo dimensões de $40 \times 70 \mathrm{~cm}$, com duas camadas, cada uma com três barras de aço de 25 $\mathrm{mm}$ de diâmetro, resultando em área de aço total na seção transversal de $30,00 \mathrm{~cm}^{2}$, como visto na Figura 7.a. Consequentemente, houve um aumento da capacidade resistente, mas afetou-se o custo total da seção, o qual teve um incremento de $4,08 \% \mathrm{em}$ razão do consumo de material.

Argolo (2000) comparou esses resultados a partir da implementação do método do Algoritmo Genético, com a análise da seção seguindo a mesma norma canadense. A seção otimizada por este método assumiu valores de $25 \times 95 \mathrm{~cm}$ para a seção transversal, reforçada por três barras de $22 \mathrm{~mm}$ de diâmetro em cada uma das duas camadas, resultando em uma área de aço de $22,81 \mathrm{~cm}^{2}$ na seção, como mostra a Figura 7.b. Isso significa uma redução de $3,56 \%$ no custo final desta seção, quando comparado ao resultado ótimo de Zielinski, Long e Troitsky (1995) e de 7,34\% em relação ao resultado prático sugerido pelos autores.

A seção ótima gerada por este estudo (Figura 7.c), segundo os critérios da norma ABNT NBR 6118 (2007) e utilizando o método Simulated Annealing para otimização da seção, apresenta uma redução de $20,67 \%$, quando comparada à seção ótima de Zielinski, Long e Troitsky (1995), de 23,78\% em relação ao resultado prático sugerido pelos autores, e em comparação à seção otimizada por Argolo (2000) a redução no custo final chega a $17,75 \%$.

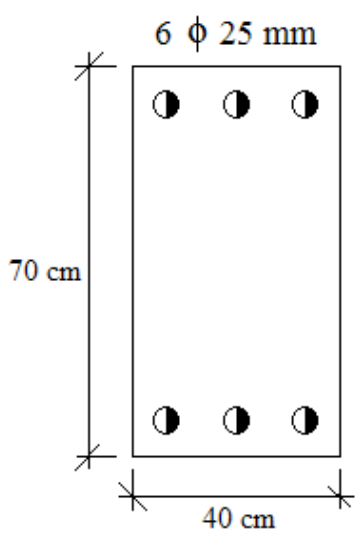

(a)

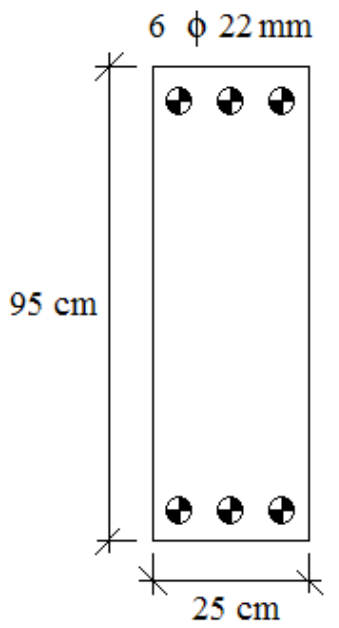

(b)

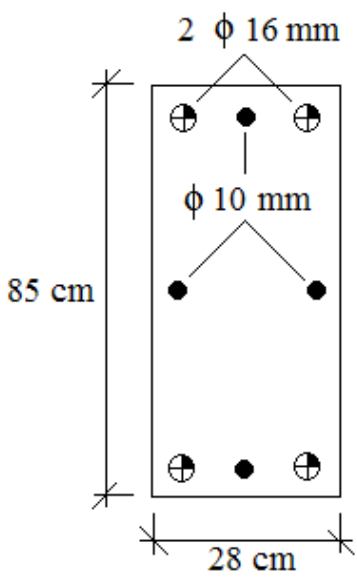

(c)

Figura 7: Detalhamento das seções do exemplo III 


\section{Conclusões}

Este trabalho tratou do problema de otimização de seções retangulares em concreto armado submetidas à flexocompressão normal, seguindo as prescrições da norma ABNT NBR 6118 (2007), empregando o método de otimização Simulated Annealing.

Com base nos resultados obtidos com a formulação implementada e feitas as comparações, foi possível concluir que o processo de otimização aqui implementado mostra-se capaz de auxiliar nas decisões e eliminar algumas das incertezas no momento de determinar os parâmetros que conduzem a projetos mais eficientes e econômicos. Neste sentido, o método Simulated Annealing mostrou-se eficiente, principalmente no tratamento das restrições impostas ao problema. Com relação especificamente ao problema estudado constatou-se que, quando comparado ao dimensionamento realizado com o auxílio de ábacos de iteração e com outras técnicas de otimização, a implementação do método Simulated Annealing demonstrou eficiência na busca por seções de menor custo, gerando soluções factíveis de execução prática. Observou-se ainda que a implementação realizada com a possibilidade de a seção ser composta por diversas bitolas de armadura contribui para a redução no custo final.

\section{Referências}

ARAÚJO, J. A. Curso de concreto armado. Rio Grande: Dunas, 2003. v. 3.

ASSOCIAÇÃO BRASILEIRA DE NORMAS TÉCNICAS. NBR 6118 - Projeto de estruturas de concreto - Procedimento. Rio de Janeiro, 2003.

NBR 8681 - Ações e segurança nas estruturas - Procedimento. Rio de Janeiro, 2004.

KIRKPATRICK, S.; GELATT, C.D.; VECCHI, M.P. Optimization by Simulated Annealing. Science, v. 220, n. 4598, p. 671-680, 1983.

KRIPKA, M. Otimização do custo de estruturas de concreto armado analisadas pelo modelo de grelha. In: SIMPÓSIO EPUSP SOBRE ESTRUTURAS DE CONCRETO, V. USP, São Paulo. 14p, 2003. CD-ROM

MARTÍNEZ MARTÍN, F. J. Optimización heurística de pilas rectangulares huecas de hormigón armado. Tese (Doutorado)__Universidad Politécnica de Valencia, Valencia, 2007.

MEDEIROS, G. F.; KRIPKA, M.

METROPOLIS, N. et al. Equation of State Calculations by Fast Computing Machines. Journal of Chem. Phys., p 1087-1092, 1953.

MONTOYA, P. J.; MESEGUER, A. G.; CABRÉ, F. M. Hormigón Armado, v. 2, 13. ed. Barcelona: Gustavo Gili, 1994.

RODRIGUES JÚNIOR, S. J. Otimização de pilares de edifícios altos de concreto armado. Tese (Doutorado em Engenharia) - Pontifícia Universidade Católica, Rio de Janeiro, 2005.

VANDERPLAATS, G. N., Numerical optimization techniques for engineering design. McGraw-Hill Book Company, 1984.

ZIELINSKI Z., A.; LONG W.; TROITSKY M. S. Designing Reinforced Concrete Short-Tied Columns Using the Optimization Technique, ACI Structural Journal, v. 92, n. 5, p. 619-625, 1995. 


\title{
Optimized dimensioning of cross-sections of reinforced concrete columns subjected to flexural compression
}

\begin{abstract}
This work presents the development and implementation of a mathematical formulation for obtaining optimal sections of reinforced concrete columns subjected to uniaxial flexural compression, based on the verification of strength proposed by the Brazilian standard NBR 6118 (ABNT 2007). To minimize the cost of the reinforced concrete columns, the Simulated Annealing optimization method was used, in which the amount and diameters of the reinforcement bars and the dimensions of the columns cross sections were considered as discrete variables. The results obtained were compared to those obtained from the conventional design procedure and other optimization methods.
\end{abstract}

Keywords: Optimization. Columns. Reinforced Concrete. Simulated Annealing.

\section{Introduction}

The search for an optimal design of an engineering structure or element that meets both performance and safety, with minimal cost and lesser environmental impact, was always the goal pursued by most structural engineers. Currently, with the advances in computer technology, it became possible to investigate a larger number of design variables and constraints, reducing the simplifications and making the mathematical model more representative of the actual state. Since there are many possible solutions, choosing the most appropriate one can be facilitated by the implementation of mathematical optimization techniques. Nowadays, there are several tools for the analysis and design of structures, but it is not common to incorporate mathematical optimization modules for these tools to dimension optimally a structure. In this context, the present paper proposes to incorporate a heuristic optimization method, the Simulated Annealing method (Kirkpatrick et al. 1983), to the process of strength verification of reinforced concrete rectangular columns subjected to uniaxial flexural compression, following the Brazilian standard NBR 6118 - Procedures for the Design of Reinforced Concrete Structures (ABNT 2007). To minimize the cost of the reinforced concrete columns, the amount and diameters of the reinforcement bars and the dimensions of the columns cross sections were considered as discrete variables. The results obtained were compared to those obtained from the conventional design procedure and other optimization methods, in an attempt to verify the influence of resistance class, variations in the magnitudes of bending moment and axial force, and material costs on the optimal design of reinforced concrete columns subjected to uniaxial flexural compression. 


\section{Problem formulation}

Considering a rectangular cross section, the objective of optimum design is to obtain a configuration that is capable of producing internal forces and moments $\left(\mathrm{N}_{\mathrm{rd}}\right.$ and $\left.\mathrm{M}_{\mathrm{rd}}\right)$ equal or higher than the applied external loadings $\left(\mathrm{N}_{\mathrm{sd}}\right.$ and $\left.\mathrm{M}_{\mathrm{sd}}\right)$, with minimal cost.

The formulation of the optimization problem starts out from the knowledge of some input parameters, previously defined and which basically represent the stresses acting on the element and the materials characteristics and costs. These design parameters do not change during the optimization process and are defined as: $\mathrm{N}_{\mathrm{sd}}-$ axial force; $\mathrm{M}_{\mathrm{sd}}$ - bending moment in relation to the axis $x$; $\mathrm{c}$ - cover depht; $\mathrm{f}_{\mathrm{yk}}-$ characteristic strength of steel; $\mathrm{E}_{\mathrm{s}}$ - elasticity modulus of steel; $\mathrm{f}_{\mathrm{ck}}$ - characteristic strength of concrete; $\mathrm{C}_{\mathrm{c}}$ - unit cost of concrete; $\mathrm{C}_{\mathrm{s}}$ - unit cost of steel; $\mathrm{C}_{\mathrm{f}}$ - unit cost of formwork.

The design variables $\left(\mathrm{x}_{\mathrm{i}}\right)$ are the values that represent the cross section dimensions and the steel bar diameters as identified in Fig. 2, where $x_{1}$ and $x_{2}$ represent, respectively, the width (b) and the height (h) of the cross section; $\mathrm{x}_{3}$ is the diameter of the four corner bars; $\mathrm{x}_{4}$ represents the number of bars in the two layers parallel to $\mathrm{x}_{1} ; \mathrm{x}_{5}$ is the diameter of the bars in the two layers parallel to $\mathrm{x}_{1} ; \mathrm{x}_{6}$ represents the number of layers with two bars parallel to $\mathrm{x}_{2}$; and $x_{7}$ is the diameter of the bars in the layers parallel to $x_{2}$.

In this study, all variables were considered as discrete, with the dimensions of the cross section varying in steps of one centimeter and the diameters of the reinforcement bars limited to those available in commercial stores.

The cost function to be minimized in the optimization process considers the total cost of materials (concrete and steel) and formwork, and can be expressed as in Eq. 12. The first term of the function represents the cost of concrete per unit volume $\left(\mathrm{C}_{\mathrm{c}}\right)$, while the second represents the cost of the longitudinal reinforcement per unit mass $\left(\mathrm{C}_{\mathrm{s}}\right)$, being $\gamma_{s}$ the specific weight of steel. The last term represents the cost of formwork per unit area $\left(\mathrm{C}_{\mathrm{f}}\right)$. All costs provide a relative value per unit length of the optimized element.

All design variables must satisfy the prescriptions of the Brazilian standard NBR 6118 (ABNT 2007) with reference to the limitations of size, spacing, and steel ratio. The constraints are listed in Eq. 13 to Eq. 23. In these equations, $e$ represents the spacing between longitudinal bars, and $\rho$ the rate of geometric reinforcement (ratio between the areas of steel and concrete sections). Regarding the constraints, a penalty function technique was adopted, in which constrained problems are transformed into unconstrained ones by adding to the function $f(x)$ a penalty function $P(x)$, which considers a multiplying factor $r$ applied to all the constraints that are not satisfied.

Overall, for the simulations performed in this work, several initial solutions were utilized, resulting in the convergence to a single solution. Regarding the optimization method, the parameters adopted were obtained from experiments and previous indication reported in the literature. 


\section{Examples}

The proposed approach, as described in previous paragraphs, was implemented from a base program previously developed by Kripka (2003) to optimize general functions with the simulated annealing method. The program was associated with a routine for checking the strength capacity of columns subjected to uniaxial flexural compression, using the FORTRAN programming language. In the next paragraphs, some examples of the numerical simulations carried out in order to test the efficiency of the proposed procedure are presented and briefly discussed. Further details can be found in Bordignon (2010).

\section{Example 1}

Argolo (2000), whose analysis was based on the Brazilian standard NBR 6118 (ABNT 1980), presented an example of uniaxial flexural compression and compared the costs for a $30 \times 70 \mathrm{~cm}$ rectangular cross section, initially dimensioned with the aid of practical iteration abacuses for three different pairs $\mathrm{N}_{\text {sd }}$ and $\mathrm{M}_{\text {sd }}$, all on the same envelope, resulting in the same area of steel for the three situations. These sections were then optimized for the longitudinal reinforcement, with fixed cross section dimensions, by using the Genetic Algorithm (GA).

The composition of the unit costs of the materials and formwork used in this example refers to March 2000 (in Brazilian Reais, $\mathrm{R} \$$ ), with the same values used by Argolo (2000), as shown in Table 1 . Likewise, the pair $\left[\mathrm{N}_{\mathrm{sd}} ; \mathrm{M}_{\mathrm{sd}}\right]$ used in this example corresponds to the second model studied by the author, since it represents a symmetrical distribution of the longitudinal reinforcement over the cross section area, similarly to the condition adopted in the present study.

From this set of conditions, two sections were generated by using the Simulated Annealing method, following the restrictions imposed by the Brazilian standard NBR 6118 (ABNT 2007): the first one with fixed dimensions of $30 \times 70 \mathrm{~cm}$, in which only the longitudinal reinforcement was optimized; and the second section, in which both the reinforcement and the cross section dimensions were optimized.

The cross section with fixed dimensions of $30 \times 70 \mathrm{~cm}$, whose reinforcement was optimized by the Simulated Annealing method, presented a cost $3.35 \%$ lower when compared to the same section optimized by the Genetic Algorithm method used by Argolo (2000). Also, when the optimal section, freely generated by the Simulated Annealing, was compared to the method used by Argolo (2000), the cost reduction increased to $6.85 \%$.

When the sections generated by the optimal Simulated Annealing method were compared to the results obtained by Argolo (2000) with the aid of practical iteration abacuses, the reduction in the total cost was $29.10 \%$ for the section with the optimized reinforcement and fixed dimensions of $30 \times 70 \mathrm{~cm}$, and $31,82 \%$ for the optimal freely generated section. 
It should be noticed, however, that the analysis performed by Argolo (2000), both by the practical method and by the optimal one, did not take into account some construction requirements established by the Brazilian standard NBR 6118 (ABNT 2007) for reinforced concrete columns. Thus, the solutions provided by the author might not be feasible from the standpoint of the formulation used in the present study.

\section{Example 2}

In this example, Zielinski et al. (1995) studied a case of uniaxial flexural compression in order to determine the cross-sectional areas of concrete and steel needed to resist the applied loads. The analysis was performed according to the Canadian standard CSA CAN3-A23.3-M84. The costs of the materials used in the example, along with the acting force and moment are presented in Table 2.

The optimum design, based on mathematical programming (MP) and using the Powell method suggested by the authors, corresponds to a rectangular cross section of $39.57 \times 68.36 \mathrm{~cm}$ and a steel section of $27.46 \mathrm{~cm}^{2}$. This section has been simplified assuming the practical dimensions of $40 \times 70 \mathrm{~cm}$, with two reinforcement layers, each one with three steel bars of $25 \mathrm{~mm}$ in diameter, resulting in a total steel section of 30.00 $\mathrm{cm}^{2}$.

Argolo (2000) compared these results to those obtained from the implementation of the Genetic Algorithm method, following the same Canadian standard. The section optimized by this method assumed values of $25 \times 95 \mathrm{~cm}$ in cross section, with three bars of $22 \mathrm{~mm}$ in diameter in each one of two reinforcement layers, resulting in a steel area of $22.8 \mathrm{~cm}^{2}$, meaning a reduction of $3.56 \%$ in the final cost of the section when compared to the optimal result obtained by Zielinski et al. (1995), and 7,34\% in relation to the practical result suggested by the same authors.

The optimal section generated by the Simulated Annealing method in the present study, following the criteria prescribed by the Brazilian standard NBR 6118 (ABNT 2007), showed a decrease in cost of 20.67\% when compared to the optimal section of Zielinski et al. (1995), 23.78\% over the practical result suggested by the same authors, and $17.75 \%$ in comparison to the section optimized by Argolo (2000).

\section{Example 3}

A problem similar to the previous one and presented by the same authors was also analyzed, but with some changes in the acting loads and in the characteristic strength of the concrete, as shown in Table 3.

Just as in the previous example, the design obtained by Zielinski et al. (1995) corresponds to a rectangular cross section with dimensions of $31.96 \times 59.36 \mathrm{~cm}$ and a total steel section of $25.80 \mathrm{~cm}^{2}$.

This section has been simplified for practical reasons, assuming dimensions of 35 x $60 \mathrm{~cm}$, with two reinforcement layers, each one with three bars of $25 \mathrm{~mm}$ in diameter, resulting in a total steel section of $30.00 \mathrm{~cm}^{2}$, raising the final cost by $9.07 \%$. 
The cross section optimized by Argolo (2000) in this example assumed dimensions of $30 \times 65 \mathrm{~cm}$, reinforced by five bars of $16 \mathrm{~mm}$ of diameter in each one of two layers, resulting in a steel section of $20.11 \mathrm{~cm}^{2}$ and leading to a cost reduction of $6.01 \%$. This reduction even reaches $13.83 \%$ when compared to the practical results suggested by the authors.

For the optimal section generated by the present study, the reduction in cost is $24.12 \%$ when compared to the optimal section, and reaches $30.43 \%$ when compared to the practical results suggested by the authors. When compared to the section optimized by Argolo (2000) the cost reduction is $19.27 \%$.

\section{Conclusions}

Based on the results obtained from the implemented formulation and comparisons performed, it was possible to conclude that the optimization process implemented showed the ability of assisting in decision making, also eliminating some of the uncertainties in determining the parameters that lead to more efficient and economic designs; in this context, the Simulated Annealing method was efficient, especially in the treatment of the constraints imposed to the problem. It was also observed that, when compared to the practical dimensioning performed with the aid of iteration abacuses and other optimization techniques, the Simulated Annealing method showed superior efficiency in the search for lower cost cross sections. In addition, the optimum cross sections proposed by the Simulated Annealing method are feasible for possible practical implementation. 